\title{
A atuação do farmacêutico na identificação e resolução de problemas relacionados a medicamentos: uma revisão integrativa
}

The role of the pharmacist in identifying and solving drug-related problems: an integrative review

El papel del farmacéutico en la identificación y resolución de problemas relacionados con las drogas: una revisión integradora

Recebido: 22/12/2021 | Revisado: 28/12/2021 | Aceito: 07/01/2022 | Publicado: 10/01/2022

\author{
Marcos Felipe Rodrigues de Souza \\ ORCID: https://orcid.org/0000-0002-6246-7525 \\ Universidade Federal do Pará, Brasil \\ E-mail: marcosfrodrigues.br@gmail.com \\ Maria Pantoja Moreira de Sena \\ ORCID: https://orcid.org/0000-0001-6641-4618 \\ Universidade Federal do Pará, Brasil \\ E-mail: mariapantojamoreira@ hotmail.com \\ Camila Martins Oliveira \\ ORCID: https://orcid.org/0000-0002-6425-2384 \\ Universidade Federal do Pará, Brasil \\ E-mail: cmofarma@gmail.com \\ Clarisse Andrade Sales \\ ORCID: https://orcid.org/0000-0001-6641-4618 \\ Universidade Federal do Pará, Brasil \\ E-mail: clarisseasales@gmail.com \\ Renato Bruno Cavalcante de Melo \\ ORCID: https://orcid.org/0000-0002-6159-3802 \\ Universidade Federal do Pará, Brasil \\ E-mail: renatobcdemelo@gmail.com \\ Crystyanne de Sousa Freitas \\ ORCID: https://orcid.org/0000-0003-4279-1587 \\ Universidade Federal do Pará, Brasil \\ E-mail: crystyannefreitas@icloud.com \\ Amanda Gabryelle Nunes Cardoso Mello \\ ORCID: https://orcid.org/0000-0001-7661-1615 \\ Universidade Federal do Pará, Brasil \\ E-mail: agncmello@gmail.com \\ Luann Wendel Pereira de Sena \\ ORCID: https://orcid.org/0000-0001-9363-5766 \\ Universidade Federal do Pará, Brasil \\ E-mail: luannsena@gmail.com
}

\begin{abstract}
Resumo
Objetivo: elaborar uma revisão integrativa quanto o papel do farmacêutico na identificação e resolução de problemas pertinentes a medicamentos, com a finalidade de criar e conduzir técnicas que intencionem aperfeiçoar a utilização segura de fármacos e favorecendo a qualidade de vida da população. Métodos: concerne a uma revisão integrativa da literatura sobre a atuação do farmacêutico na identificação e resolução de problemas relacionados a medicamentos. Resultados: as falhas de medicação compõem um problema de saúde pública mundial, pois além dos males provocados à saúde do paciente, produzem aumento da internação hospitalar, acréscimo nos custos de saúde e contribuem para os entraves financeiros dirigidos a este setor. Em maior parte, os erros podem ser evitáveis, podendo ser realizado tanto por profissionais novos como pelos experientes, sejam médicos, farmacêuticos, enfermeiros, técnicos, cuidadores e o próprio paciente. Conclusão: $\mathrm{O}$ farmacêutico é um profissional substancial na equipe de saúde designada ao cuidado de pacientes que estão em uso de medicamentos, tendo de agir de forma integrada com os demais profissionais, com o objetivo único de possibilitar a saúde e sustentar um tratamento eficaz para o paciente. O profissional configura uma das últimas viabilidades de discernir, reparar ou limitar prováveis riscos associados à terapia.

Palavras-chave: PRM; Farmacêutico; Farmácia clínica.
\end{abstract}




\begin{abstract}
Objective: to develop an integrative review of the role of the pharmacist in identifying and solving problems related to medications, with the aim of creating and conducting techniques that intend to improve the safe use of drugs and favoring the population's quality of life. Methods: concerns an integrative literature review on the role of pharmacists in identifying and solving drug-related problems. Results: medication failures are a global public health problem, as in addition to the harm caused to the patient's health, they produce an increase in hospital stays, increase in health costs, and contribute to the financial barriers directed at this sector. For the most part, errors are preventable and can be performed by both new and experienced professionals, whether doctors, pharmacists, nurses, technicians, caregivers, and the patient. Conclusion: The pharmacist is a substantial professional in the health team assigned to the care of patients who are using medication, having to act in an integrated manner with other professionals, with the sole objective of enabling health and sustaining an effective treatment for the patient. The professional is one of the last possibilities to discern, repair or limit probable risks associated with the therapy.
\end{abstract}

Keywords: PRM; Pharmacist; Clinical pharmacy.

\title{
Resumen
}

Objetivo: desarrollar una revisión integradora del rol del farmacéutico en la identificación y resolución de problemas relacionados con los medicamentos, con el objetivo de crear y ejecutar técnicas que pretendan mejorar el uso seguro de los medicamentos y favorecer la calidad de vida de la población. Métodos: se refiere a una revisión integradora de la literatura sobre el papel de los farmacéuticos en la identificación y resolución de problemas relacionados con las drogas. Resultados: medicación son un problema de salud pública global, ya que además del daño causado a la salud del paciente, producen un aumento de las estancias hospitalarias, aumento de los costos de salud y contribuyen a las barreras financieras dirigidas a este sector. En su mayor parte, los errores se pueden prevenir y pueden ser realizados tanto por profesionales nuevos como experimentados, ya sean médicos, farmacéuticos, enfermeras, técnicos, cuidadores y el paciente. Conclusión: El farmacéutico es un profesional sustancial en el equipo de salud asignado a la atención de los pacientes que están usando medicación, debiendo actuar de manera integrada con otros profesionales, con el único objetivo de posibilitar la salud y sustentar un tratamiento eficaz para el paciente. El profesional es una de las últimas posibilidades para discernir, reparar o limitar los probables riesgos asociados a la terapia.

Palabras clave: PMR; Farmacêutico; Farmacia clínica.

\section{Introdução}

O uso de medicamentos é a forma mais habitual de tratamento em nossa sociedade. Ainda que, ocorram esforços em prol da utilização racional de medicamentos (URM), há dados que comprovam a presença de problemas de saúde cuja início esteja ligado aos fármacos. As influências sociais às quais estão sujeitos os prescritores, a ordenação do sistema de saúde e o marketing farmacêutico são comumente citados como razões implicados nesta problemática (Aquino et al., 2020).

Usualmente, o emprego da farmacoterapia predomina-se somente na efetividade e não na segurança. De fato, examinar a garantia dos medicamentos requer não só um controle e seguimento estrito dos pacientes, para identificar cedo as reações adversas, mas também, reconhecer e avaliar interações medicamentosas, o cumprimento do tratamento e os erros da medicação (Melo et al., 2020).

A utilização inadequada de medicamento é capaz de exibir ocorrências que são julgadas como Problemas Relacionados a Medicamentos (PRM). Os PRM são estabelecidos como uma adversidade de saúde, com efeitos clínicos negativos decorrentes da farmacoterapia que, realizados por várias razões, direcionam a não obtenção dos propósitos terapêuticos ou ao advento de efeitos não desejados (Abdin et al., 2020). O gasto anual dos PRM está ente 30 e 130 bilhões de dólares e a presença de um programa de cuidado farmacêutico conseguiria restringir em cerca de 45,6 bilhões de dólares as despesas de cuidados médicos diretos (Rocha et al., 2020).

Tendo em consideração que, os PRM em várias ocasiões não são presentes no decorrer do diagnóstico, essa descrição conduziu a uma atuação assistencial efetiva do farmacêutico. A partir de então, este profissional teria como um de seus propósitos, rastrear a provável associação entre queixa ou problema de saúde do paciente e/ou medicamento. Além de detectálos, auxiliaria na solução destes problemas, colaborando para contenção da morbimortalidade e aumento a qualidade de vida da população. 
Neste sentido, este estudo teve como propósito produzir uma revisão integrativa acerca do papel do farmacêutico na identificação e resolução de PRM, com o intuito de criar e conduzir técnicas que intencionem aperfeiçoar a utilização segura de medicamentos, contribuindo para a qualidade de vida da população brasileira (França et al., 2021).

\section{Metodologia}

Refere-se a uma revisão integrativa da literatura sobre a atuação do farmacêutico na identificação e resolução de PRM (Mendes et al., 2019). A seleção dos estudos foi executada nas bases de dados Google Scholar, Scientific Electronic, Library Online (SciELO), Medical Literature Analysis and Retrieval System Online (MEDLINE) e Literatura Latino-americana e do Caribe em Ciência da Saúde (LILACS), por meio dos descritores: "problemas relacionado a medicamentos" e "farmacêutico", nos idiomas português e inglês.

Os padrões de adição determinados para separação dos artigos foram: artigos que difundiam texto completo disponível nos idiomas português, inglês e espanhol, segundo o tema apresentado, com livre acesso e anunciado nos últimos cinco anos. Os artigos de revisão e publicações superiores a cinco anos foram excluídos.

Para a preparação da amostra, foi efetuada a averiguação dos artigos de acordo com os fatores de inclusão, seguida por seleção fundamentada na literatura dos títulos e resumos, com posterior exclusão dos estudos que não se enquadrassem na temática da revisão ou que ficassem duplicados nas bases de dados.

Logo após, foi realizada a análise dos artigos elegíveis com base na leitura integral do seu conteúdo, excluindo-se os artigos não adequados para essa revisão, com posterior seleção final da amostra (Figura 1).

Figura 1 - Processo de seleção dos estudos.

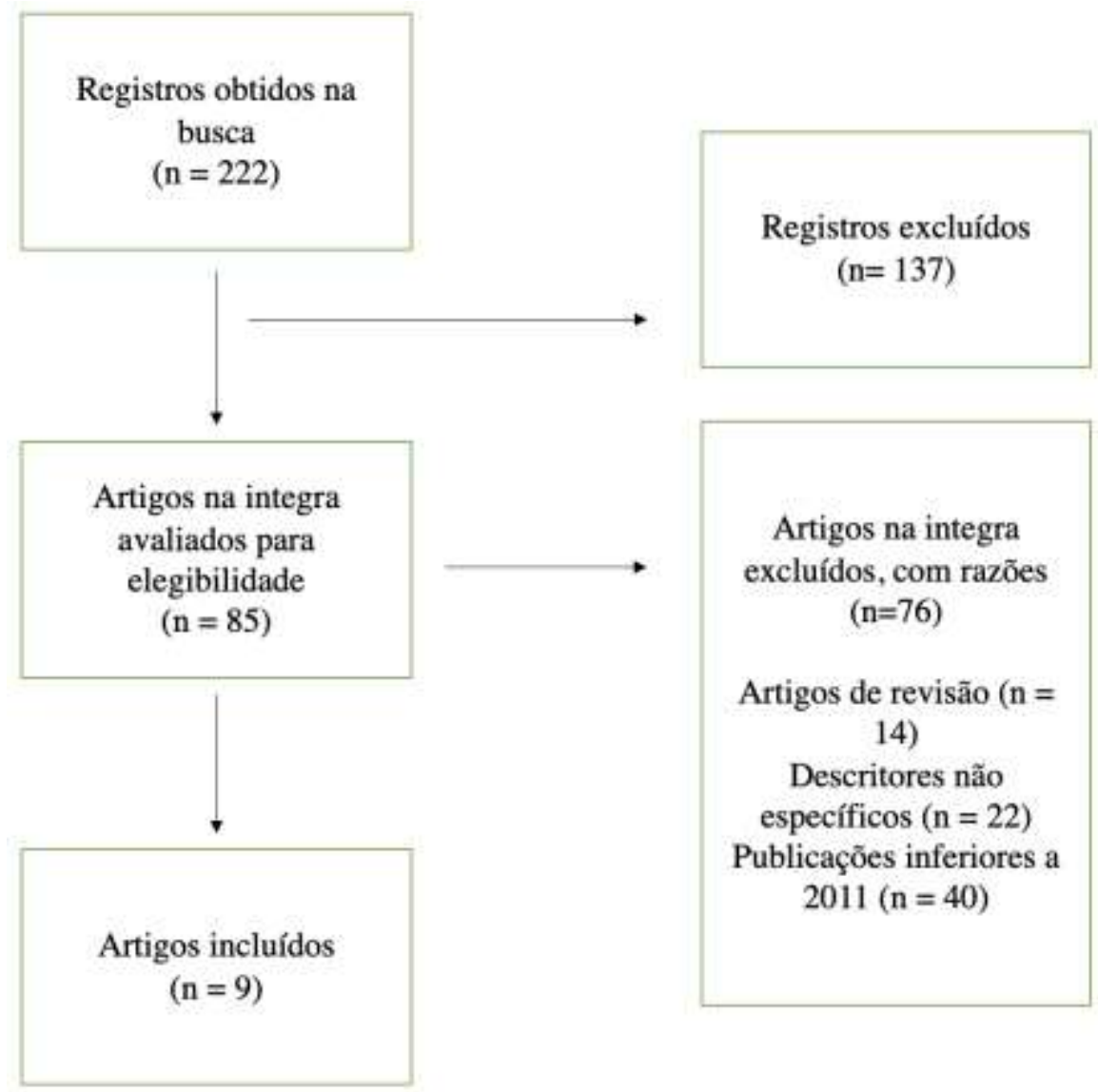

Fonte: Autores. 


\section{Resultados}

O meio de opção dos estudos localiza-se na Figura 1. Apareceram 222 artigos eventualmente importantes. Destes, 137 foram excluídos na fase de triagem. Foram obtidos e lidos na totalidade 85 artigos, das quais 14 foram excluídos por ser artigo de revisão, 22 por não possuírem descritos estabelecidos e 40 devido ao tempo de publicado, inferior a onze anos. No final, permaneceram elegíveis 9 artigos científicos (Tabela 1).

Tabela 1 - Descrição dos estudos selecionados.

\begin{tabular}{|c|c|c|c|c|}
\hline $\mathbf{N}^{\mathbf{0}}$ & Autor (ano)/ Periódico & Título & Tipo de Estudo & Objetivos \\
\hline 1 & $\begin{array}{ll}\text { Frick et al. (2010) / } \\
\text { Revista } & \text { Contexto } \\
\text { Saúde } & \end{array}$ & $\begin{array}{c}\text { Principais problemas encontrados } \\
\text { nas prescrições em município da } \\
\text { região noroeste do estado do Rio } \\
\text { Grande do Sul }\end{array}$ & Descritivo observacional & $\begin{array}{c}\text { Identificar e analisar os erros mais } \\
\text { frequentes presentes nas prescrições } \\
\text { médicas }\end{array}$ \\
\hline 2 & $\begin{array}{l}\text { Aizentein et al (2011) / } \\
\text { Revista de Ciências } \\
\text { Farmacêuticas Básica } \\
\text { e Aplicada }\end{array}$ & \begin{tabular}{|c|} 
Problemas relacionados a \\
medicamentos; reações adversas a \\
medicamentos e erros de \\
medicação: a necessidade de uma \\
padronização nas definições e \\
classificações \\
\end{tabular} & Descritivo observacional & $\begin{array}{l}\text { Elaborar uma proposta de termos } \\
\text { relacionados aos problemas } \\
\text { relacionados a medicamentos }\end{array}$ \\
\hline 3 & $\begin{array}{l}\text { Carvalho et al. (2013) / } \\
\text { Revista Eletrônica de } \\
\text { Farmácia }\end{array}$ & $\begin{array}{l}\text { Investigação de problemas } \\
\text { relacionados com medicamentos } \\
\text { em uma instituição para longa } \\
\text { permanência para idosos }\end{array}$ & $\begin{array}{l}\text { Exploratório descritivo com } \\
\text { abordagem quantitativa }\end{array}$ & $\begin{array}{c}\text { Identificar os principais problemas } \\
\text { relacionados aos medicamentos, as } \\
\text { patologias mais frequentes e as } \\
\text { classes farmacológicas mais } \\
\text { prescritas entre os idoso } \\
\end{array}$ \\
\hline 4 & $\begin{array}{l}\text { Gerlack et al. (2015) / } \\
\text { Revista Brasileira de } \\
\text { Farmácia Hospitalar }\end{array}$ & $\begin{array}{c}\text { Problemas relacionados ao uso de } \\
\text { medicamentos em idosos } \\
\text { atendidos em ambulatório } \\
\text { multiprofissional de hospital } \\
\text { universitário no Rio Grande do Sul }\end{array}$ & $\begin{array}{l}\text { Estudo de prevalência } \\
\text { retrospectivo de caráter } \\
\text { descritivo e analítico }\end{array}$ & \begin{tabular}{|c|} 
Descrever os problemas \\
relacionados ao uso de \\
medicamentos identificados a partir \\
do acompanhamento \\
farmacoterapêutico de idosos \\
atendidos em ambulatório \\
\end{tabular} \\
\hline 5 & $\begin{array}{l}\text { Paes et al. (2018) / } \\
\text { Revista Científica da } \\
\text { FMC }\end{array}$ & $\begin{array}{c}\text { Identificação dos problemas } \\
\text { relacionados a medicamentos } \\
\text { antimicrobianos em pacientes } \\
\text { internados na clínica médica de } \\
\text { um hospital escola } \\
\end{array}$ & Descritivo observacional & $\begin{array}{c}\text { Identificar problemas relacionados a } \\
\text { medicamentos antimicrobianos }\end{array}$ \\
\hline 6 & $\begin{array}{l}\text { Souza et al. (2018) / } \\
\text { Pensar Acadêmico }\end{array}$ & $\begin{array}{c}\text { Importância do farmacêutico } \\
\text { clínico no uso seguro e racional de } \\
\text { medicamentos no âmbito } \\
\text { hospitalar }\end{array}$ & $\begin{array}{l}\text { Estudo descritivo } \\
\text { retrospectivo }\end{array}$ & $\begin{array}{l}\text { Analisar se prática clínica do } \\
\text { farmacêutica favorece a adesão } \\
\text { terapêutica do paciente }\end{array}$ \\
\hline 7 & $\begin{array}{l}\text { Resende et al. (2019) / } \\
\text { Einstein }\end{array}$ & $\begin{array}{c}\text { Problemas relacionados ao uso de } \\
\text { medicamentos em pacientes com } \\
\text { tuberculose e HIV/AIDS em } \\
\text { hospital de referência }\end{array}$ & Estudo transversal & $\begin{array}{c}\text { Determinar a frequência de } \\
\text { problemas relacionados ao uso de } \\
\text { medicamentos no tratamento de } \\
\text { pacientes com tuberculose e } \\
\text { HIV/AIDS } \\
\end{array}$ \\
\hline 8 & $\begin{array}{l}\text { Junior et al. (2021) / } \\
\text { Revista Saúde em Foco }\end{array}$ & $\begin{array}{c}\text { Importância da farmácia clínica } \\
\text { para a identificação e resolução de } \\
\text { problemas relacionados a } \\
\text { medicamentos (PRM) }\end{array}$ & Descritivo observacional & $\begin{array}{c}\text { Determinar a Importância da } \\
\text { farmácia clínica para a identificação } \\
\text { e resolução de problemas } \\
\text { relacionados a medicamentos (PRM) }\end{array}$ \\
\hline 9 & $\begin{array}{l}\text { Paula et al. (2021) / } \\
\text { Brazilian Journal of } \\
\text { Development }\end{array}$ & $\begin{array}{c}\text { Uso irracional de medicamentos: } \\
\text { uma perspectiva cultural }\end{array}$ & Descritivo observacional & $\begin{array}{c}\text { Identificar os motivos do uso } \\
\text { irracional de medicamentos: uma } \\
\text { perspectiva cultural }\end{array}$ \\
\hline
\end{tabular}

Fonte: Autores.

As falhas de medicação compõem um problema de saúde pública mundial, pois além dos males provocados à saúde do paciente, produzem aumento da internação hospitalar, acréscimo nos custos de saúde e contribuem para os entraves financeiros dirigidos a este setor. 
Frick et al (2010), ao estudar os notáveis problemas vistos nas prescrições da região noroeste do estado do Rio Grande do Sul, percebeu que 43,33\% possuíam erros, tais como: inexistência de carimbo médico, nome incompleto do paciente, os medicamentos não se encontravam de acordo com denominação comum brasileira (DCB), sem posologia e outros.

De acordo com Aizenstein et al. (2011), os erros podem estar associados aos mecanismos e sistemas da realidade profissional que envolvem: a prescrição, informação de pedido, rotulagem, dispensação, distribuição, administração e adesão do paciente. Em maior parte, as falhas podem ser evitáveis, podendo ser realizado tanto por profissionais novos como pelos experientes, sejam médicos, farmacêuticos, enfermeiros, técnicos, cuidadores e o próprio paciente. O mesmo autor diz que, os medicamentos detêm um papel expressivo no cuidado, manutenção e melhora da saúde e auxiliam para a melhoria das características de vida da população. Todavia, apesar dos seus benefícios a prescrição e a aplicação inadequada de medicamentos, formam uma das principais razões de distúrbios à saúde e perdas econômicas na população (Aizenstein et al., 2011).

O predomínio de doenças crônicas e o consumo de fármacos, ampliam a ocorrência dos PRM, gerando e/ou potencializando atenuação da ação de inúmeros meios fisiológicos, incluindo os referentes à cinética e a dinâmica dos medicamentos. Freitas et al. (2013), ao pesquisar os PRM em um instituto para longa estadia para idosos, notou que, nesta faixa etária, os PRM mais percebidos foram o emprego de drogas contraindicados e a elevada interações medicamentosas, atribuído pela existência de polifarmácia na localidade.

Em concordância com as respostas achadas por Freitas et al. (2013), Gerlack et al. (2015), ao explorar esta mesma faixa etária viu que as interações medicamentosas, as possibilidades de efeitos adversos e a administração incorreta, foram os transtornos mais constantes, respectivamente.

De outro ponto de vista, o consumo descontrolado de medicamentos, dentre eles os antimicrobianos, pode originar relevantes efeitos como reações adversas, acréscimo da resistência bacteriana e despesas irrelevantes. Paes et al. (2018) afirma que os antimicrobianos são uma classe cuja prescrição é constante na clínica médica e que seus principais PRM, está referente as interações medicamentosas consideráveis, reações adversas e erros de omissão de cuidado.

Souza et al (2018) enfatiza que o farmacêutico é um profissional fundamental na equipe de saúde indicada ao cuidado de pacientes que estão em uso de medicamentos. Este tem de agir de forma ajustada com os demais profissionais (médicos, fisioterapeutas, enfermeiros, biomédicos, nutricionistas, outros) com o propósito de oportunizar a saúde e garantia de um tratamento eficaz aos pacientes.

Os farmacêuticos, como componentes da equipe multiprofissional de cuidado aos pacientes, auxiliam para análise das questões relativas à dose dos medicamentos, interações medicamentosas, omissão e frequência indevida da terapia, melhorando os resultados clínicos (Rezende et al., 2019).

De acordo com Junior et al. (2021) os ofícios de farmácia clínica (SFC) encontra-se em avanço no Brasil e todos os PRM vistos, torna-se claro a precisão de integrar o farmacêutico clínico junto às equipes de saúde, visto que, a ocorrência de PRM é alarmante e as intervenções do profissional podem constituir proveitos diretos para a proteção do paciente, bem como, possibilitar a melhoria na qualidade do cuidado e redução de custos.

Neste sentido, o uso irracional de medicamentos (URM) reflete um desafio global associado a saúde, com consequências significativas tanto para o indivíduo, sistema de saúde e sociedade como um todo. Levando em considerando que diversas causas podem viabilizar, em distintos estágios e ciclos de uso, o entendimento desses fatores são essenciais para alterar a conduta profissional e/ou da população e possibilitar o consumo racional de medicamentos (Paula et al. 2021). 


\section{Discussão}

Promover o acesso a medicamentos de qualidade eficiente tem sido uma inquietação mundial. Neste caso, o desenvolvimento da atividade farmacêutica, através da filosofia do cuidado, aparece conjuntamente à demanda de determinar o aumento da morbimortalidade relativa aos fármacos. Assim, o profissional precisa se reorientar, pois dispõe de capacidades para aprimorar os resultados terapêuticos e a qualidade de vida dos pacientes dentro dos meios disponíveis (Anderson et al., 2019).

Os serviços farmacêuticos (SF) dirigidos ao cuidado ao paciente tem sido relacionado aos avanços dos resultados da saúde, com restrição dos efeitos adversos relacionados a medicamentos e da morbimortalidade, auxiliando para a melhoria da qualidade de vida dos pacientes, além de uma redução nos custos econômicos usados com cuidados em saúde (Barberato et al., 2019).

Dentre as atribuições do farmacêutico, a análise da prescrição é uma ação indiscutível e primordial para dispensação de medicamento, pois objetiva atestar a proteção e o êxito terapêutico do paciente. Apesar de, a prescrição de medicamentos responder a distintas legislações, conforme com as especificidades de cada setor da saúde e disposição técnica do profissional, o procedimento tem fases similares que compreendem o reconhecimento das necessidades de saúde do paciente, estabelecimento das finalidades terapêuticas, orientação ao paciente, prescrição e avaliação dos resultados (Soares et al., 2020).

Embora seja impedido ao farmacêutico alterar a prescrição de medicamentos do paciente, dita por outro prescritor, o profissional ao longo da dispensação precisa analisar se a prescrição cumpre os critérios básicos de indicação, posologia e contraindicações (Anderson et al., 2019). Em âmbitos hospitalares, a avaliação da prescrição vem sendo empregue como instrumento planejado para conceituar o trinfo terapêutico e certificar a farmacoterapia com segurança e qualidade. Entretanto, os farmacêuticos que exercem suas atividades em farmácias comunitárias, igualmente contribuem para um padrão de saúde ligado no reconhecimento de erros de prescrição e no cuidado farmacêutico de qualidade. Os profissionais asseguram modos de impedir PRM e explicações sobre o seu uso correto com a finalidade de expandir as possibilidades do desfecho terapêutico (Rocha et al., 2021).

Nos últimos anos, a aflição com a segurança na utilização de medicamentos tem se otimizado devido a farmacoterapia dispor de um papel expressivo no tratamento, prevenção, diagnóstico e controle de doenças. Segundo estudos, por volta de 5\% a $6 \%$ das hospitalizações estão associadas ao uso impróprio de fármacos, bem como, mais da metade dessas internações seriam evitáveis. Os erros de medicação (EM) acontecem quando o medicamento está sob responsabilidade do profissional de saúde, do paciente ou do consumidor, e por isso, podem estar correlacionados tanto a prescrição, quanto à dispensação ou administração do medicamento (Botelho et al., 2017).

A resolução do Conselho Federal de Farmácia (CFF) nº 585 de 29 de agosto de 2013, que instaura as prerrogativas do farmacêutico clínico, dispõe sobre a atenção à prescrição e afins. Ela determina a ação do farmacêutico na organização e avaliação da farmacoterapia, análise da prescrição quanto as questões legais e técnicas, intervenções com o intuito de auxiliar na farmacoterapia, acesso ao prontuário, prevenção e identificação de qualquer PRM (CFF, 2013; Maioli et al., 2018). Acredita-se que, para tentar impedir os PRM, o profissional necessita levar em consideração três fatores: o paciente, a análise da prescrição em si com a intervenção, se necessário, e por último o acompanhamento do paciente pós-intervenção. Neste sentido, ações voltadas a programas educacionais e tecnologia de informação podem ser tomadas com o objetivo de evitar os erros de prescrição e/ou PRM, porém, o farmacêutico clínico agindo por meio do cuidado à prescrição, tem se revelado o meio mais eficiente na prevenção de internações pelo uso de medicamentos (Santos et al., 2019).

Dentre as classes de medicamentos que requerem cautela devidos problemas que podem produzir, temos os antimicrobianos (Araújo et al., 2017). O uso incorreto dos antibióticos e o não cumprimento da prescrição são condições 
fundamentais para a resistência bacteriana. A realização de programas de cuidado farmacêutico poderá promover o estudo do perfil de consumo de antimicrobianos e a realização de intervenções educativas junto aos pacientes e prescritores, resultando em uma redução da prescrição e utilização irracional destes medicamentos. Como produto, a redução de PRM é considerável, limitando os agravos à saúde dos indivíduos e consequentemente gastos econômicos (Carvalho et al., 2017).

Logo, os empenhos para a readequação de atividades e práticas profissionais, visando o uso racional dos medicamentos (URM) é fundamental numa sociedade em que os fármacos constituem um conjunto terapêutico mais utilizado (Dias et al., 2019). O farmacêutico precisa ter comportamento e habilidades que propiciem integrar-se à equipe de saúde e interagir mais com os pacientes e a comunidade, ajudando para a melhoria da qualidade de vida, em especial, no que se refere à otimização da farmacoterapia e ao URM (França et al., 2021).

Contudo, os atos do farmacêutico, no modelo de cuidado em saúde, na maioria das vezes, são clínicos individuais. Mas, as regularizações das intervenções farmacêuticas e a troca de ideias com outros profissionais de saúde pode favorecer um efeito no nível coletivo e na promoção do uso seguro e racional de medicamentos e, consequentemente, diminuição dos PRM, ajudando assim, o individuo a obter melhores resultantes durante a farmacoterapia (Guimarães et al., 2021).

\section{Considerações Finais}

O farmacêutico é um profissional substancial na equipe de saúde designada ao cuidado de pacientes que estão em uso de medicamentos, tendo de agir de forma integrada com os demais profissionais, com o objetivo único de possibilitar a saúde e sustentar um tratamento eficaz para o paciente. O profissional configura uma das últimas viabilidades de discernir, reparar ou limitar prováveis riscos associados à terapia.

Para um domínio maior, recomenda-se que seja exibido nos próximos estudos, as consequências qualitativas e quantitativas dessas práticas na saúde, tencionando sempre a promoção de educação, melhor acesso e adesão ao tratamento.

\section{Referências}

Abdin, M. S., Grenier, G. L., \& Guénette, L. (2020). Impact of pharmacists' interventions on the pharmacotherapy of patients with complex needs monitored in multidisciplinary primary care teams. International Journal of Pharmacy Practice, 28 (1): 75-83.

Aizenstein, M. L., \& Tomassi, M. H. (2011). Problemas relacionados a medicamentos; reações adversas a medicamentos e erros de medicação: a necessidade de uma padronização nas definições e classificações. Revista de Ciências Farmacêuticas Básica Aplicada, 32 (2): $169-173$.

Anderson, S. L., Marrs, J. C., \& Vande, G. J. P., Hanratty, R. (2019). Implementation of a Clinical Pharmacy Specialist-Managed Telephonic Hospital Discharge Follow-Up Program in a Patient-Centered Medical Home. Population Health Management, 16 (4): 235-241.

Aquino, D. S., Barros, J. A. C., \& Silva, M. D. P. (2020). A automedicação e os acadêmicos da área de saúde. Ciências Saúde Coletiva, 15 (5): $2533-2538$.

Araújo, P. S., Costa, E. A., Junior, A. A. G., Acurcio, F. A., Guibu, I. A., Álvares, J., Costa, K. S., Karnikowski, M. G. O., Soeiro, O. M., \& Leite, S. N. (2017). Atividades farmacêuticas de natureza clínica na atenção básica no Brasil. Revista de Saúde Pública, 51 Supl 2:6s.

Barberato, L. C., Scherer, M. D. D. A., \& Lacourt, R. M. C. (2019). The pharmacist in the Brazilian Primary Health Care: insertion under construction. Ciências \& Saúde Coletiva, 24 (10): 3717-3726.

Botelho, J. A., \& Roese, F. M. (2017). Intervenções Realizadas Pelo Farmacêutico Em Uma Unidade De Pronto Atendimento Médico. Revista Brasileira de Farmácia Hospitalar e Serviços de Saúde, 8 (1): 34-36.

Carvalho, D. M. O., Rocha, R. M. M., \& Freitas, R. (2013). Investigação de problemas relacionados com medicamentos em uma instituição para longa permanência para idosos. Revista Eletrônica de Farmácia, 10 (2): 24-41.

Carvalho, M. N., Álvarez, J., Costa, K. S., Junior, A. A. G., Acurcio, F.A., Costa, E. A., Guibu, I. A., Soeiro, O. M., Karnikowski, M. G. O., \& Leite, S. N. (2017). Força de trabalho na assistência farmacêutica da atenção básica do SUS, Brasil. Revista de Saúde Pública, 51 Supl 2: 16 s.

Conselho Federal de Farmácia. [Resolução nº 585 de 29 de agosto de 2013: Regulates clinical assignments pharmacist and makes other arrangements].

Dias, D., Wiese, L. P. L., Pereira, E. M., \& Fernandes, F. M. (2019). Avaliação de intervenções clínicas farmacêuticas em uma UTI de um Hospital Público de Santa Catarina. Revista Brasileira de Farmácia Hospitalar e Serviços de Saúde, 9 (3): 1-5. 
Research, Society and Development, v. 11, n. 1, e41811125053, 2022

(CC BY 4.0) | ISSN 2525-3409 | DOI: http://dx.doi.org/10.33448/rsd-v11i1.25053

França, C., \& Andrade, L. G. (2021). Performance of the Pharmacist in health care in community Pharmacies. Revista Ibero-Americana de Humanidades, Ciência e Educação, 7 (9): 10-20.

Frick, G. G. G., Bonotto, L. F., Bernardi, C. L. B., \& Pletsch, M. U. (2010). Principais problemas encontrados nas prescrições em municípios da região noroeste do estado do Rio Grande do Sul. Revista Contexto \& Saúde, 10 (19): 108-111.

Gerlack, L. F., Werlang, M. C., \& Bós, A. J. G. (2015). Problemas relacionados ao uso de medicamentos em idosos atendidos em ambulatório multiprofissional de hospital universitário no Rio Grande do Sul. Revista Brasileira de Farmácia Hospitalar e Serviços de Saúde, 6 (1): $13-17$.

Guimarães, P. H. D., Pacheco, R. P., \& Morais, Y. J. (2021). Pharmaceutical care and the use of Over-the-Counter Medications (MIPs). Research, Society and Development, 10 (12): e485101220405.

Júnior, L. A. B. A., Leite, R. S., Yoshida, E. H., Estanagel, T. H. P., \& Santos, N. S. (2021). Importância da farmácia clínica para a identificação e resolução de problemas relacionados a medicamentos (PRM). Revista Saúde em Foco, 13 (1):9-20.

Maioli, N. A., \& Santos, H. C. B. (2018). Intervenções Farmacêuticas e sua importância na Segurança do Paciente Hospitalizado. Colloq Vitae, 10 (2): 35-40.

Melo, R. C., \& Pauferro, M. R. V. (2020). Educação em saúde para a promoção do uso racional de medicamentos e as contribuições do farmacêutico neste contexto. Brazilian Journal of Development, 6 (5): 32162-32173.

Mendes, K. D. S., Silveira, R. C. C. P., \& Galvão, C. M. (2019). Uso de gerenciador de referências bibliográficas na seleção dos estudos primários em revisão interativa. Revista Texto e Contexto Enfermagem, 28 (1): e20170204.

Paes, G. C. S., \& Ferreira, C. E. F. (2018). Identificação dos problemas relacionados a medicamentos antimicrobianos em pacientes internados na clínica médica de um hospital escola. Revista Científica da FMC, 13 (1): 30-35.

Paula, C. C. S., Campos, R. B. F., \& Souza, M. C. R. F. (2021). Uso irracional de medicamentos: uma perspectiva cultura. Brazilian Journal of Development, 7 (3): 21660-21676.

Resende, N. H., Miranda, S. S., Ceccato, M. G. B., Haddad, J. P. A., Reis, A. M. M., Silva, D. I., \& Carvalho, W. S. (2019). Problemas relacionados ao uso de medicamentos em pacientes com tuberculose e HIV/AIDS em hospital referência. Einstein, 17 (4): 1-9.

Rocha, H. M. S. G., Silva, V. G., Vieira, A. P. B. F., Tavares, M. L. D., Pinheiro, P. N. Q., \& Andrade, M. A. (2020). Consulta farmacêutica como estratégia para redução de problemas relacionados à farmacoterapia: revista sistemática. Brazilian Journal of Development, 6 (12): $97838-97855$.

Santos, N. S., Marengo, L. L., Moraes F. S., \& Barberato. (2019). Intervenções para reduzir a prescrição de medicamentos inapropriados para idosos. Revista de Saúde Pública, 53:7.

Soares, L. S. S., Brito. E. S., \& Galato, D. (2020). Percepções de atores sociais sobre Assistência Farmacêutica na atenção primária: a lacuna do cuidado farmacêutico. Saúde em Debate, 44 (1): 411-426.

Souza, L. B., Souza, D. M., Souza, S. M., Silva, D. R., \& Aguilar, N. C. (2018). Importância do farmacêutico clínico no uso seguro e racional de medicamentos no âmbito hospitalar. Pensar Acadêmico, 16 (1): 109-124. 